ECCOMAS

Proceedia
COMPDYN 2021

$8^{\text {th }}$ ECCOMAS Thematic Conference on Computational Methods in Structural Dynamics and Earthquake Engineering

M. Papadrakakis, M. Fragiadakis (eds.) Streamed from Athens, Greece, 28 - 30 June 2021

\title{
PERSPECTIVES ON THE ACCELERATION OF THE NUMERICAL ASSESSMENT OF FLUTTER AND BUFFETING RESPONSE OF BRIDGE DECKS
}

\author{
Julien Heremans $^{1,2}$, Anass Mayou ${ }^{1}$, and Vincent Denoë ${ }^{1}$ \\ ${ }^{1}$ Structural \& Stochastic Dynamics, University of Lige, Belgium \\ Alle de la Dcouverte, 1, B-4000 Lige Belgium \\ e-mail: julien.heremans@uliege.be \\ ${ }^{2}$ F. R. S.-FNRS, National Fund for Scientific Research, Belgium \\ Rue d'Egmont, 8, B-1000 Bruxelles, Belgium
}

\begin{abstract}
The critical flutter speed of a bridge deck is the result of an eigen value analysis. However, the progressive growth of the response for sub-critical wind velocities, resulting from the buffeting action of the turbulent wind, is also of major concern to the designers. The complete flutter analysis of a bridge structure therefore requires the repeated analysis of the aeroelastic response for various wind velocities, starting from zero (wind off) to the critical flutter speed. In a spectral approach, each of these analyses is typically based on the heavy integration of the power spectral density of the aeroelastic response. It has been recently found that this integration can be facilitated by a background/resonant decomposition as is commonly implemented in a buffeting-only setting. The paper describes a preliminary work showing that this decomposition can be extended to the flutter-and-buffeting configuration. The work is only preliminary since it deals with a single oscillator. However, it shows a massive CPU saving of one to two orders of magnitude, while limiting the error to one percent. This kind of saving is particularly expected to make affordable the analysis of large multi degree-of-freedom structures.
\end{abstract}

Keywords: Multiple Timescale Spectral Analysis, Background, Resonant.

ISSN:2623-3347 (C) 2021 The Authors. Published by Eccomas Proceedia.

Peer-review under responsibility of the organizing committee of COMPDYN 2021.

doi: $10.7712 / 120121.8860 .18700$ 


\section{INTRODUCTION}

The critical flutter speed of a bridge deck corresponds to an aeroelastic instability of the coupled system composed of the bridge and the surrounding air flow [1,2,3]. This instability is recognized as potentially very harmful since the famous collapse of the Tacoma-Narrows bridge [4]. Codes and standards [5] take a significant safety margin with respect to this critical wind speed and typically do not allow to overcome $80 \%$ of this critical velocity.

Besides, the progressive growth of the response for sub-critical wind velocities, resulting from the buffeting action of the turbulent wind, is also of major concern to the designers [6, 7].The complete flutter analysis of a bridge structure therefore requires the repeated analysis of the aeroelastic response for various wind velocities, starting from zero (wind off) to the critical flutter speed, or at least to the design wind speed [8]. In a spectral approach, each of these analyses is typically based on the time-consuming integration of the power spectral density (PSD) of the aeroelastic response.

Traditional integration methods struggle to efficiently estimate these integrals because of the significant peakedness of the function in the neighborhood of the natural frequencies. In this paper, we present an extension of the Background/Resonant decomposition (which is commonly applied under the quasi-steady assumption), to aeroelastic analysis, where the stiffness and damping of the coupled system changes with frequency. The study is limited to single degree-of-freedom systems at this stage but constitutes the cornerstone of an extension to multi degree-of-freedom systems, where such an approximation becomes very interesting in terms of computational efficiency.

In the following sections, the mathematical background is presented, then the algorithmic implementation is briefly discussed and the method is illustrated with a few examples. Finally, the perspectives of application to multi degree-of-freedom (MDOF) structures are discussed.

\section{EXTENSION OF THE BACKGROUND/RESONANT DECOMPOSITION TO THE AEROELASTIC OSCILLATOR}

The dynamics of a single degree-of-freedom (SDOF) system subjected to buffeting and aerodynamic loads is governed by

$$
m_{\mathrm{s}} \ddot{q}(t)+c_{\mathrm{s}} \dot{q}(t)+k_{\mathrm{s}} q(t)=f_{\mathrm{bu}}(t)+f_{\mathrm{ae}}(t),
$$

where $m_{\mathrm{s}}, c_{\mathrm{s}}$ and $k_{\mathrm{s}}$ are the mass, viscosity and stiffness of the structural system, and where $t$ is the time. The loading consists of a buffeting loading $f_{\mathrm{bu}}(t)$ which is here assumed to be characterized as a Gaussian stochastic process and a so-called unsteady aerodynamic loading $f_{\text {ae }}(t)$, which is usually expressed as a convolution of the structural response $(q(t), \dot{q}(t))$ with aeroelastic indicial functions. In the frequency domain, $F_{\mathrm{ae}}(\omega)=\left[\mathrm{i} \omega c_{\mathrm{ae}}(\omega)+k_{\mathrm{ae}}(\omega)\right] Q(\omega)$ and the governing equation becomes

$$
\left[-m_{\mathrm{s}} \omega^{2}+\mathrm{i} \omega c(\omega)+k(\omega)\right] Q(\omega)=F_{\mathrm{bu}}(\omega),
$$

where $c(\omega)=c_{\mathrm{s}}-c_{\mathrm{ae}}(\omega)$ and $k(\omega)=k_{\mathrm{s}}-k_{\mathrm{ae}}(\omega)$ gather both the structural and aerodynamic viscosity and stiffness. The later ones can be expressed as a function of Theodorsen's function (flat plate, [9]) or, more generally, as Scanlan's derivatives (e.g. bridge deck [1]).

The power spectral density of the structural response $q(t)$ is thus obtained by

$$
S_{q}(\omega ; U)=\left|\left[-m_{\mathrm{s}} \omega^{2}+\mathrm{i} \omega c(\omega)+k(\omega)\right]^{-1}\right|^{2} S_{f, \mathrm{bu}}(\omega),
$$


and the variance of the response is ultimately obtained by integration of (3),

$$
\sigma_{q}^{2}(U)=\int_{-\infty}^{+\infty} S_{q}(\omega ; U) \mathrm{d} \omega
$$

In a structural design process, this integral has to be computed for several values of the wind velocity $U$, which enters in the modeling through $c_{\mathrm{ae}}(\omega)$ and $k_{\mathrm{ae}}(\omega)$ which are expressed as a function of the reduced frequency $K=\omega B / U$ where $B$ is a characteristic size of the structural element (deck width). Standard integration schemes based on the trapezoidal rule or GaussLobatto integration schemes [10] do provide an accurate result, they might seem too slow for application in large scale problems or when the bridge stability has to be assessed in a probabilistic manner, which requires many repetitions of the deterministic problem, see Section 5

In the wind engineering community, this type of integral is also encountered in the buffetingonly analysis of structures, i.e. without unsteady forces. The method, implemented in the community by Davenport [11, 12], based on the existing approaches in aeronautics [13], has resulted in the well known Background/Resonant (B/R) decomposition. The use of multiple timescales in the computation of the wind induced response of structures in not new. It has already been used to determine the statistics of non Gaussian responses [14] or of nonlinear aerodynamic loading terms [15]. The general method, based on stretches and rescaling of the frequency bands contributing to the integral has been generalized under the terminology Multiple Timescales Spectral Analysis (MTSA) [16].

In the present configuration, under the following assumptions:

- the timescales of the loading and of the system are significantly different. In other words, a distinction is made between the slow dynamics represented by the buffeting loading, and fast dynamics represented by the natural vibrations of the aeroelastic system. The centroid of the power spectral density of the buffeting load shall be substantially lower ( 5 to 10 times lower) than the natural frequency of the aeroelastic system;

- the structural damping ratio is small, smaller than or of the same order of magnitude as $5 \%-10 \%$; the quality of the approximation worsens as the damping ratio grows beyond these values;

- the frequency dependent stiffness and damping $k(\omega)$ and $c(\omega)$ vary smoothly and moderately in the neighborhood of the resonance peak of the aeroelastic system,

it is actually possible to demonstrate [17], by application of the general concepts of the Multiple Timescale Spectral Analysis [16], that the variance is expressed as a sum of the background and the resonant contributions

$$
\sigma_{q}^{2}=\sigma_{q, \mathrm{~B}}^{2}+\sigma_{q, \mathrm{R}}^{2}
$$

where

$$
\sigma_{q, \mathrm{~B}}^{2}=\int_{-\infty}^{+\infty} \frac{S_{f, \mathrm{bu}}(\omega)}{\left(k_{\mathrm{s}}-k_{\mathrm{ae}}(\omega)\right)^{2}} \mathrm{~d} \omega \quad ; \quad \sigma_{q, \mathrm{R}}^{2}=\frac{S_{f, \mathrm{bu}}(\bar{\omega})}{\left(k_{\mathrm{s}}-k_{\mathrm{ae}}(\bar{\omega})\right)^{2}} \frac{\pi \bar{\omega}}{2 \bar{\xi}} \frac{1}{1+\frac{1}{2} \frac{\bar{\omega} \partial_{\omega} k_{\mathrm{ae}}(\bar{\omega})}{k_{\mathrm{s}}-k_{\mathrm{ae}}(\bar{\omega})}} .
$$

They are readily interpreted as the aeroelastic extensions of the well known decomposition introduced by Davenport. In this expression $\bar{\omega}$ represents the resonance frequency of the aeroelastic system; it is defined by

$$
\frac{\bar{\omega}^{2}}{\omega_{\mathrm{s}}^{2}}=\frac{k_{\mathrm{s}}-k_{\mathrm{ae}}(\bar{\omega})}{k_{\mathrm{s}}} .
$$


In wind-off conditions, $k_{\mathrm{ae}}(\bar{\omega} ; U=0)=0$ and $\bar{\omega}=\omega_{\mathrm{s}}$ is therefore equal to the natural structural frequency. As the wind velocity increases, the natural frequency of the aeroelastic system $\bar{\omega}$ might drift more or less significantly from $\omega_{\mathrm{s}}$ depending on the specificities of the aerodynamic stiffness $k_{\mathrm{ae}}(\omega)$.

\section{ALGORITHMIC IMPLEMENTATION}

This section describes a practical implementation of the algorithm. At this stage, the implemented model is suitable for SDOF models only but its extension to MDOF models is also discussed.

\subsection{Algorithm for SDOF structures}

The flow-chart of the solution strategy is shown in Figure 1. For a given average wind velocity, the solving process consists of 3 main tasks: the evaluation of the aeroelastic eigen frequency, and that of the background and resonant components. The determination of the eigen frequency of the aerodynamic system relies on an iterative scheme, initiated with the wind-off conditions. Relaxation can be used to improve the convergence of the process. As the wind-off conditions give already an excellent guess of the natural frequency of the aeroelastic system, the convergence is quite straightforward. The resonant component is then evaluated using (6), and Scanlan's formulation for $k_{\mathrm{ae}}(\omega), c_{\mathrm{ae}}(\omega)$ and $\partial_{\omega} k_{\mathrm{ae}}(\omega)$. The last step is the determination of the background component. Depending on the specificities of $k_{\mathrm{ae}}(\omega)$ and the targeted accuracy, the integration of the buffeting forces according to (6) can be simplified. Indeed, in cases where the background component plays a secondary role, $\sigma_{q, \mathrm{~B}} \ll \sigma_{q, \mathrm{R}}$, its accurate determination is no longer a necessity and the denominator in the expression for $\sigma_{q, \mathrm{~B}}$ in (6) can be simply replaced by $k_{\mathrm{s}}^{2}$. This leads to a drastic simplification of the computation of the integral since $\sigma_{q, \mathrm{~B}}$ then boils down to the usual quasi-static formulation of Davenport. When this assumption can be made - and this is what is done in the rest of this paper-, the background component does not require any integration point, the resonant component just requires one integration point (located at the natural frequency) and the proposed formulation, referred to as MTSA in the sequel, is just based on a single integration point.

All this solving process is embedded in a loop over a range of $U$, from wind-off to critical conditions.

\subsection{Extension to MDOF structures}

Up to now, the use of the background/resonant decomposition for aeroelastic systems was only discussed on SDOF models. For such simple models, the computational load is proportional to the number of wind velocities considered in the analysis. Since the analysis for each wind velocity is rapid, there is no big issue and the process can be repeated for a comfortable number of wind velocities, which allows an accurate evaluation of the transition towards instability on approaching the critical wind velocity. In the case of MDOF models, every single run for a given velocity might turn to be much more expensive. Indeed, if the number of degrees of freedom is large, a very time-consuming operation appears in the algorithm: the projection of the buffeting forces in the modal basis. This is explained by considering that, in a standard linearized model of the buffeting forces, the wind force at each degree-of-freedom is expressed as a linear transformation of the local wind velocity, i.e.

$$
\mathbf{F}_{\mathrm{bu}}(t)=\mathbf{A u}(t)+\mathbf{b}
$$




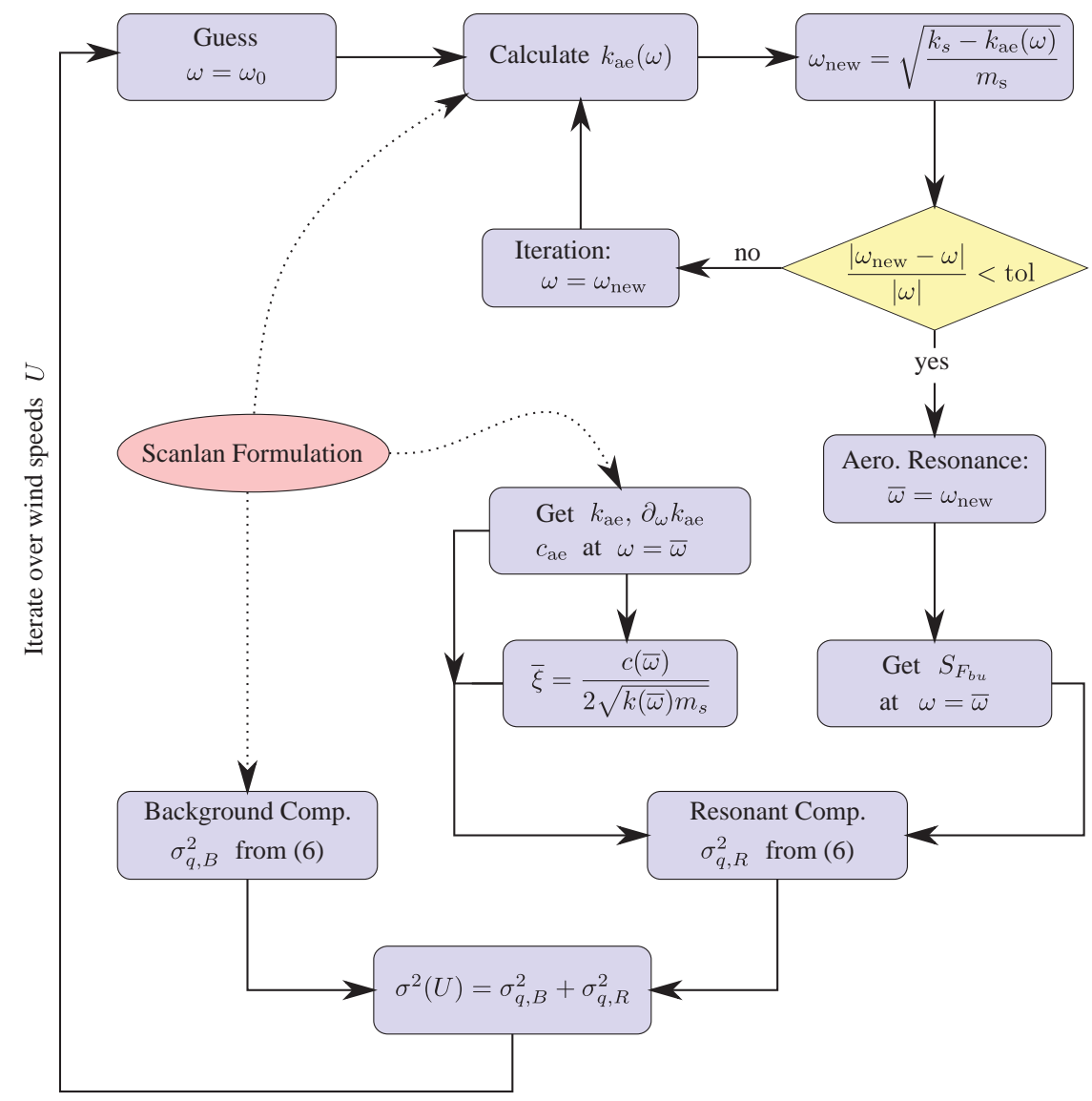

Figure 1: Organigram of the aeroelastic analysis performed with the background/resonant decomposition for evaluating the integral of the response spectra. The solving process is embedded in a loop over the average wind speeds.

where $\mathbf{u}(t)$ is a column vector containing the fluctuating component of the wind velocities at the nodes of the finite element model, the power spectral density of the buffeting load projected in mode $i$ reads

$$
S_{F_{\mathrm{bu}}^{*}}^{i}(\omega)=\boldsymbol{\phi}_{i}^{T} \mathbf{A S}_{\mathbf{u}} \mathbf{A}^{T} \boldsymbol{\phi}_{i} .
$$

The evaluation of the PSD of the modal forces, including their cross-PSD, is particularly time consuming since it involves the left- and right-multiplication by large matrices.

In short, the computational time for the modal analysis of a large MDOF structure is mostly conditioned by the number of frequencies where the PSD must be sampled, i.e. the number of projections such as (9). As a result, the determination of the variance as the quadrature of the PSD of the structural response (in each mode) is the operation that requires being optimally implemented. Three solutions are studied in the rest of this paper to determine these integrals with various levels of smartness : (i) first, a classical trapezoidal method is implemented; it requires typically a large number of uniformly distributed points to accurately capture the sharp peaks of the spectra, (ii) an adaptative integration scheme is also considered, which is an interesting solution to lighten the computational burden by optimizing the integration mesh, distributing a (smaller) number of points at well chosen locations, (iii) third, a method of integration based on the proposed background/resonant decomposition.

The development of the background/resonant decomposition for spectral aerodynamic analysis is quite straightforward, at least if the modal response is uncoupled. In the latter case, the 
analysis is performed mode by mode, and the SDOF model is still applicable provided that the mass, damping and stiffness are replaced by their modal homologue. Cases of structures experiencing modal coupling require however slightly more advanced models [23], but remains in the scope of the approximation that will constitutes the core of the future investigations.

In the next section, these integration methods with uniform and non uniform meshes will be compared with the background/resonant decomposition. A special attention will be paid to the trade-off between number of integration points and accuracy of the results.

\section{ILLUSTRATIONS AND EVALUATION OF CPU LOAD SAVING}

In this section, the efficiency of the background/resonant decomposition is illustrated on two realistic case studies, namely the Golden Gate bridge and Tacoma Narrows bridge. The aeroelastic behavior of these two structures is characterized by torsional flutter as governing failure mode. The structure behavior is idealized by a SDOF model governed by

$$
\left(-\omega^{2} I_{s}+\mathrm{i} \omega\left(c-c_{\mathrm{ae}}(\omega)\right)+k-k_{\mathrm{ae}}(\omega)\right) \Theta(\omega)=M_{\mathrm{b}}(\omega)
$$

where $I_{s}$ is the mass moment of inertia per unit length and such that the aeroelastic damping, stiffness, and the buffeting pitching moment are reduced to

$$
\begin{aligned}
& M_{\mathrm{bu}}(\omega)=\frac{q B}{U}\left(\frac{\pi}{2} B A(\omega)\right) W(\omega) \\
& k_{\mathrm{ae}}(\omega)=q B^{2}\left(\frac{\omega B}{U}\right)^{2} A_{3}^{*}(\omega) \\
& c_{\mathrm{ae}}(\omega)=q B^{2} \frac{B}{U}\left(\frac{\omega B}{U}\right) A_{2}^{*}(\omega) .
\end{aligned}
$$

The $A_{2}^{*}(\omega)$ and $A_{3}^{*}(\omega)$ coefficients refer to the Scanlan's - or flutter- torsional derivatives (see Figure 2), the admittence $A(\omega)$ is supposed to be constant and equal to 1 in the following illustrations and $W(\omega)$ is the spectral representation of the vertical fluctuating component of the wind turbulence. The structural parameters are summarized in Figure 1. For the two test cases, the accuracy and the efficiency of the proposed background/resonant decomposition will be compared to classical integration methods. The trapezoidal scheme is standard, while the adaptative integration scheme is briefly detailed in the Appendix. In each case, the integral in (4) is computed where $S_{q}(\omega ; U)$ corresponds to $S_{\theta}(\omega ; U)$, for these case of torsional flutter. It is also defined by (3) where the aeroelastic stiffness and viscosity are given by (11).

As previously discussed, the number of integration points has a direct impact on the calculation load, and consequently on the computation time: the smaller the number of integration points, the faster the wind flutter analysis. The CPU times are not reported for this simple SDOF structure; they are replaced by the number of integration points which is assumed to give an correct picture of the computational burden for larger problems.

\subsection{Golden Gate bridge}

Figure 3-a shows the PSD of the torsional degree-of-freedom for a selection of wind velocities. As the wind velocity increases, the resonance peak is shifted to the left and becomes sharper. This is a sign of torsional flutter, i.e. aero-elastic instability by lack of damping. The integral of the PSDs obtained for various wind velocities, i.e. the variance, is used to determine the scaled standard deviation of the angular displacement $\theta$ shown in Figure 3-b. Each 


\begin{tabular}{lll}
\hline & Golden Gate & Tacoma \\
\hline \hline Moment of Inertia $I_{s}\left[\mathrm{~kg} \cdot \mathrm{m}^{2} / \mathrm{m}\right]$ & $4.4 \cdot 10^{6}$ & $177.73 \cdot 10^{3}$ \\
Natural frequency $f_{s}[\mathrm{~Hz}]$ & 0.1916 & 0.20 \\
Damping Ratio $\xi_{s}[\%]$ & 0.5 & 0.5 \\
Deck width $B[\mathrm{~m}]$ & 27.43 & 12.0 \\
\hline \hline
\end{tabular}

Table 1: Structural properties of the structures considered in Illustrations 1 and 2.

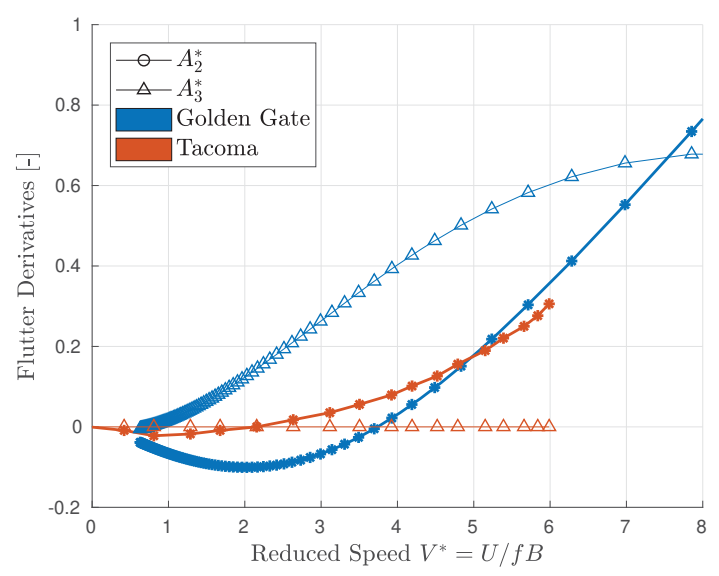

Figure 2: Flutter derivatives $A_{2}^{*}(\omega)$ and $A_{3}^{*}(\omega)$ of the considered structures .

point of the reference curve was obtained with the trapezoidal integration scheme with a large number $(50,000)$ of integration points. Red dots represents the results obtained with the $B / R$ decomposition while the individual components $\mathrm{B}$ and $\mathrm{R}$ are also represented to indicate that the instability occurs for this type of cross-section by means of the resonant component. The reference solution and the proposed approximation are in excellent agreement, promising a very low relative error. As shown in Figure 3-c, the damping ratio remains constantly lower than $1 \%$ for all wind velocities, indicating that the hypothesis formulated above related to small damping is respected.

In Figure 3-d, the number of integration points required to satisfy a given relative precision is represented for the two usual numerical methods (trapezoidal scheme and adaptative integration) as well as the proposed Multiple Timescale Spectral Analysis approach (background/resonant decomposition). The relative error is calculated with respect to a reference solution obtained from a classical trapezoidal integration method with a mesh of 50,000 points uniformly spaced between 0 and $10 \mathrm{~Hz}$ (i.e. $62.8 \mathrm{rad} / \mathrm{s}$ ). This mesh was kept constant for all considered wind speeds.

The square symbols, associated with the trapezoidal scheme, indicate that this classical integration method requires a minimum of 10,000 integration points to get a relative error of the order of the percent pour all wind speeds. For 1,000 points and below, the error is already way too large and the function is not accurately represented. However, with 1,000 points smartly distributed using an adaptive integration method, the standard deviation is accurately calculated with an error lower than $0.01 \%$. A good compromise can be found around 100 points, where the error is of the order of the percent again. This method reduces the number of integration points required by a factor 100, approximatively. The performance of this adaptive integration method 


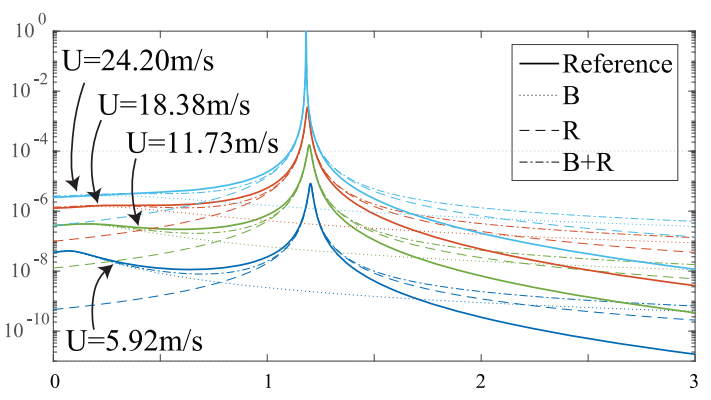

(a) Circular Frequency $[\mathrm{rad} / \mathrm{s}]$

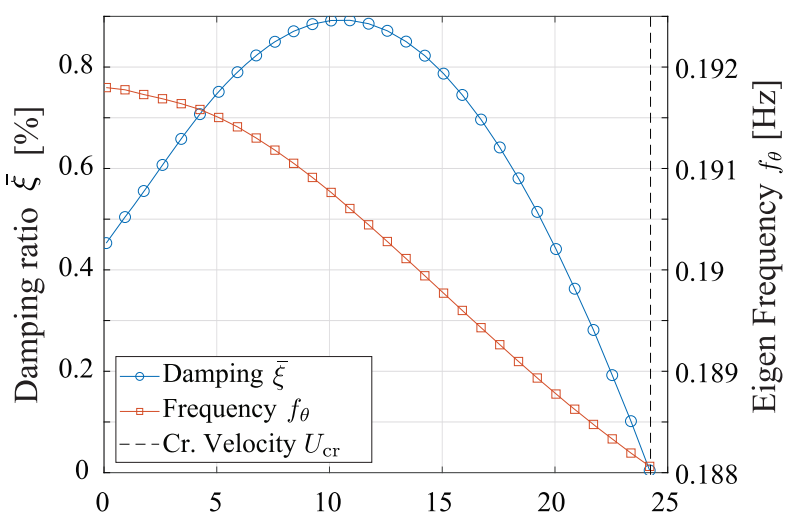

(c)

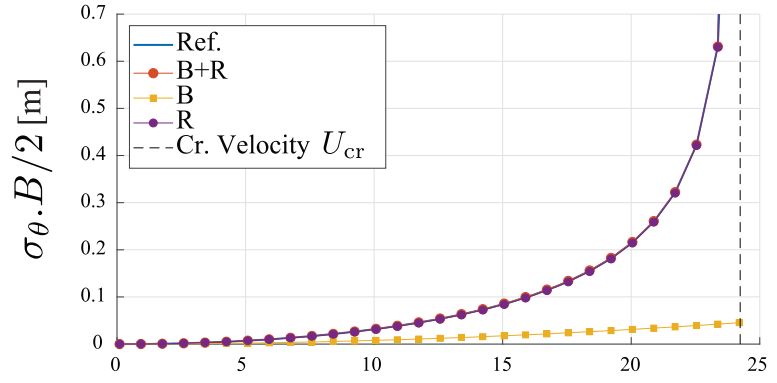

(b)

Wind Velocity $U[\mathrm{~m} / \mathrm{s}]$

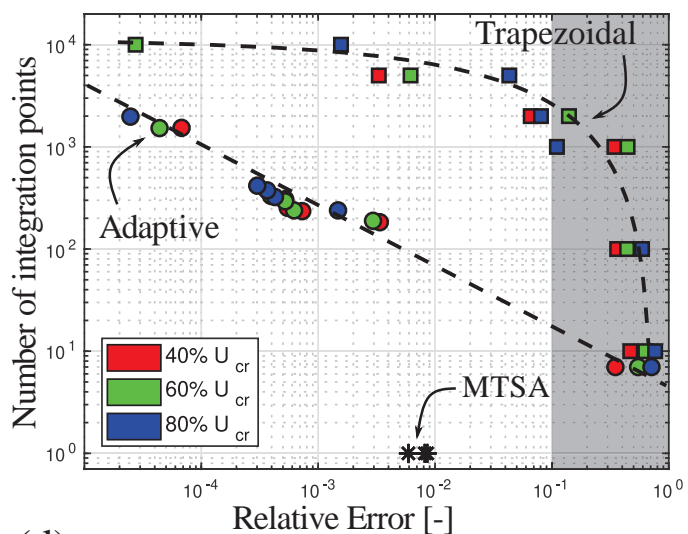

(d)

Figure 3: Aerodynamics response under buffeting loads (Golden Gate bridge). (a) PSD and their B and R approximations for different wind velocities. (b) Scaled standard deviations obtained with accurate numerical integration (Reference), background component (B), resonant component (R), and the sum of them (B)+(R). (c) Damping ratio and natural frequency at resonance with respect to avg. wind velocity. (e) Number of integration points required to satisfy a given relative error on $\sigma_{\theta}$ for 3 avg. wind velocities and for the three methods discussed (trapezoidal scheme with uniform sampling, adaptive quadrature and the MTSA based B/R decomposition .)

is of course conditioned by the efficiency of the refinement algorithm and also by the parameters passed by the user - for instance the initial mesh. Usage of other adaptive integration scheme than the algorithm presented in the appendix might therefore lead to similar trends but slightly different numbers. The last cluster of points represented in Figure 3-d refers to the B/R decomposition and suggests an error below $1 \%$ while being limited a single sampling point, at resonance. This arises from the white noise approximation made in (6). This method appears now to be therefore the best compromise number of points/accuracy.

\subsection{Tacoma Narrows bridge}

The second case study is the Tacoma Narrows bridge. Besides its dangerously low critical velocity of $11 \mathrm{~m} / \mathrm{s}$, it behaves very similarly to the Golden Gate bridge such that most of results are globally the same. The output resulting from the aeroelastic analysis are shown in Figure 4.

For this second application the damping related flutter derivative $A_{2}^{*}(\omega)$ is such that the damping ratio at resonance $\bar{\omega}$ diminishes as $U$ increases, except at very low wind speeds. The damping ratio remains again below $1 \%$ which explains why the quality of the approximation does not drop as the wind velocity increases. The trapezoidal integration scheme, in contrast, fails to capture the sharp resonance peak on approaching the critical flutter speed. More than that, the proposed background/resonant decomposition is even more precise that the damping ratio is small. Because the damping drops as the considered wind velocity approaches the crit- 


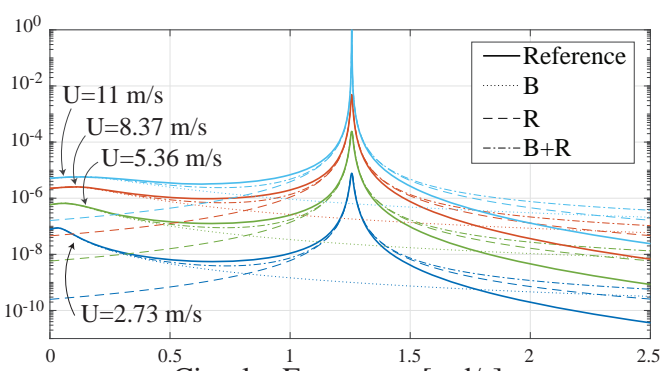

(a)

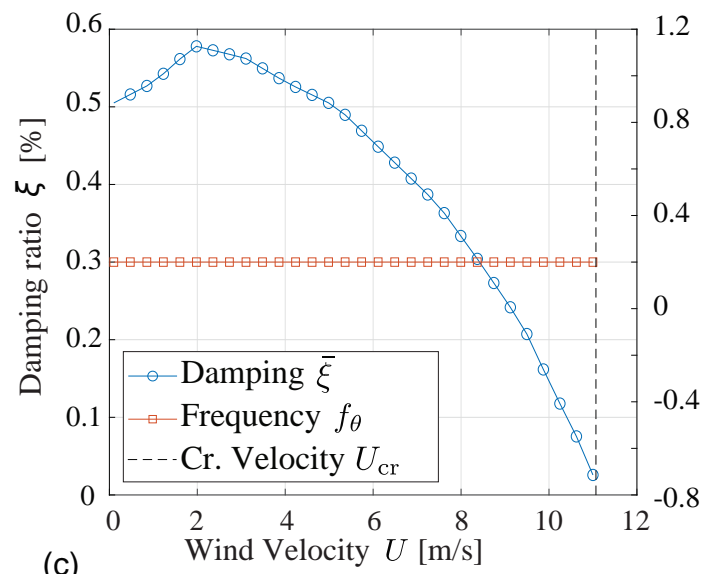

(c)

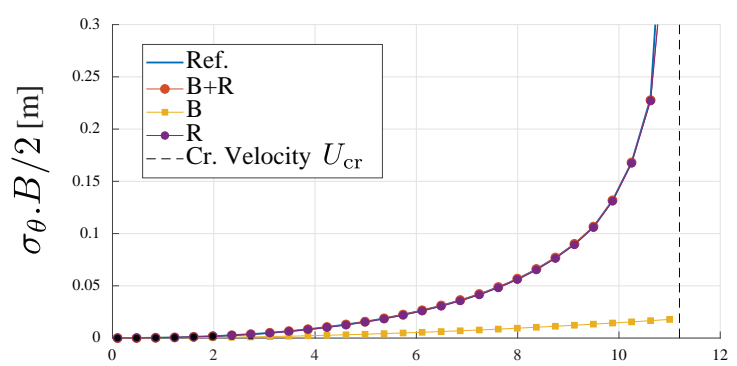

(b)

Wind Velocity $U[\mathrm{~m} / \mathrm{s}]$

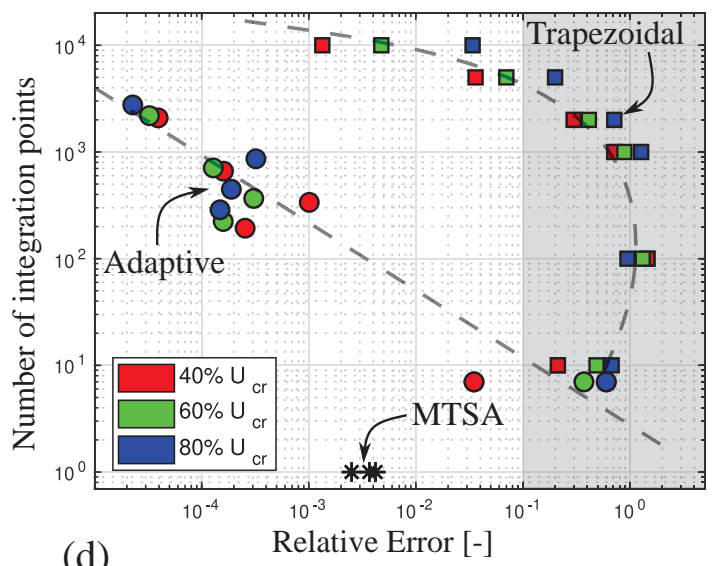

(d)

Figure 4: Aerodynamics response under buffeting loads (Tacoma Narrows bridge). (a) PSD and their B and R approximations for different wind velocities. (b) Scaled standard deviations obtained with accurate numerical integration (Reference), background component (B), resonant component (R), and the sum of them (B)+(R). (c) Damping ratio and natural frequency at resonance with respect to avg. wind velocity. (e) Number of integration points required to satisfy a given relative error on $\sigma_{\theta}$ for $3 \mathrm{avg}$. wind velocities and for the three methods discussed (trapezoidal scheme with uniform sampling, adaptive quadrature and the MTSA based B/R decomposition .)

ical velocity, the approximation is even more accurate and appealing as flutter conditions are close. This is unfortunately not applicable to divergence instabilities, where the loss of stiffness is typically accompanied by an increase in damping, see e.g. XXX (bench polimi). Therefore, the approximation should not be recommended for divergence instabilities, particularly close to the flutter conditions.

Regarding the relative error and the integration points, the same 3 tendencies as in the previous applications are observed. A number of 100 integration points are required to accurately integrate the PSD using an adaptive method, and 10.000 points at least should be used if a uniform mesh is used. The MTSA approach provides truly accurate standard deviation with an error lower than $0.5 \%$, slightly lower than in the Golden Gate bridge illustration.

\section{PERSPECTIVES}

The acceleration of the computation of the aeroelastic response of a single degree-of-freedom system could seem ridiculous at the time where the computational power is continuously growing and especially when the multiple mode analysis of large structures has been being available for more than 20 years $[18,19,20]$. We see however two main promising perspectives for this preliminary work.

The first one is immediate and concerns the stochastic stability of the single degree-offreedom system. Indeed in a probabilistic approach, should it be by means of Monte Carlo simulations [21] or alternatives such as the collocation method or Galerkin approache [22], it is 
essential to repeat the solution of the deterministic problem a large amount of times. Any CPU saving is therefore welcome to determine more accurate statistics of the response.

A second perspective of application of the proposed formulation concerns the extension to multi degree-of-freedom systems. They are governed by an equation similar to (1), which reads, in the frequency domain,

$$
\left[-\mathbf{M}_{\mathrm{s}} \omega^{2}+\mathrm{i} \omega \mathbf{C}(\omega)+\mathbf{K}(\omega)\right] \mathbf{Q}(\omega)=\mathbf{F}_{\mathrm{bu}}(\omega) .
$$

The aeroelastic matrices $\mathbf{M}_{\mathrm{s}}, \mathbf{C}$ and $\mathbf{K}$ combining the structural matrices and aerodynamic unsteady loads might be very large. An analysis in the modal basis is able to drastically decrease the size of the system. The variance of the modal responses in each mode can then be determined with the method proposed in this paper. The main difference with a classical integration of the power spectral densities is that the projection of the buffeting forces in the modal basis is performed for natural frequencies only — or for a very restricted number of points in the most unfavorable case of a large aeroelastic stiffness-, and not for the whole set of natural frequencies that a Gauss-Lobatto or trapezoidal integration scheme would require. So the proposed method can be used with very little adaptations as long as modal responses are concerned. Then, the combination of modal responses is necessary to determine structural responses (displacements and internal forces) or, alternatively, equivalent static loads. This requires the estimation of modal correlation coefficients but again, the Multiple Timescale Spectral Analysis has already been used to solve this problem in the buffeting-only case [23] and it is believed that the same extension is possible. Depending on how the eigenmodes of the aeroelastic system are determined, a proper recourse to the smallness of off-diagonal terms might be invoked, similarly to some of the previous results of the authors [24]. All in all, the determination of the response of a single oscillator appears as a major building block for the derivation of a very fast algorithm for the analysis of the aeroelastic response of large bridges. The outlook for future is to make possible the probabilistic analysis of large-scale structures or the rapid parametric analysis of large scale structures (varying the wind incidence, optimization of the aerodynamic deck, etc.).

\section{CONCLUSIONS}

The analysis of an aeroelastic oscillator subjected to low frequency turbulence can be studied by means of background and resonant components, which take a more general form than in the well known case presented by Davenport. They remain simple, however, and offer therefore a straightforward understanding of the response. Beyond this gain in physical insight, the derivation of the two components in an appropriate algorithmic implementation allows to speed up the determination of the stochastic response.

Examples have shown that the number of sampling points is reduced by a factor 10.000 (!) with respect to classical integration method, and by a factor 100 with respect to a smarter adaptive integration technique. This promises massive computational time saving, especially since the projection of the nodal aerodynamic loads vector in modal basis is by far the exhausting operation of the algorithm.

The delicacy of the proposed approximation resides also in the quality of its predictions: in the studied examples, the error committed on the standard deviation is lower than $1 \%$ which remains quite accurate when put in perspective to the safety envelope usually taken in civil engineering applications. The approximation method assumes that the damping ratio is small, and this hypothesis is even more respected that the system is close to flutter (except (static) divergence). Therefore the prediction is even more accurate that the resonant peaks are sharp, 
as opposed to the trapezoidal integration method with a uniform mesh which struggles to capture accurately the sharpness of the peaks with a reasonable number of points.

These performance are promising for future extensions to probabilistic contexts, or large multiple mode structures, that rely both entirely on the efficiency of the algorithm to be envisaged.

\section{REFERENCES}

[1] Robert H Scanlan and J Tomo. Air foil and bridge deck flutter derivatives. Journal of Soil Mechanics \& Foundations Div, 1971.

[2] Robert H Scanlan and Nicholas P Jones. Aeroelastic analysis of cable-stayed bridges. Journal of Structural Engineering, 116(2):279-297, 1990.

[3] Robert H Scanlan. Problematics in formulation of wind-force models for bridge decks. Journal of Engineering Mechanics, 119(7):1353-1375, 1993.

[4] Peter A Irwin, Stoyan Stoyanoff, Jiming Xie, and Mark Hunter. Tacoma narrows 50 years later?wind engineering investigations for parallel bridges. Bridge structures, 1(1):3-17, 2005 .

[5] EN. EN 1991-1-1 Eurocode 1: Actions on Structures - Part 1-4: Wind actions, Brussels, 2005. CEN.

[6] Tajammal Abbas, Igor Kavrakov, and Guido Morgenthal. Methods for flutter stability analysis of long-span bridges: a review. In Proceedings of the Institution of Civil Engineers-Bridge Engineering, volume 170, pages 271-310. Thomas Telford Ltd, 2017.

[7] Giorgio Diana, Stoyan Stoyanoff, Ketil Aas-Jakobsen, Andrew Allsop, Michael Andersen, Tommaso Argentini, Miguel Cid Montoya, Santiago Hernandez, Jose Angel Jurado, Hiroshi Katsuchi, Igor Kavrakov, Ho-Kyung Kim, Guy Larose, Allan Larsen, Guido Morgenthal, Ole Oiseth, Simone Omarini, Daniele Rocchi, Martin Svendsen, and Teng Wu. Iabse task group 3.1 benchmark results. part 1: Numerical analysis of a two-degree-offreedom bridge deck section based on analytical aerodynamics. Structural Engineering International, 0(0):1-10, 2019.

[8] Ahsan Kareem and Yukio Tamura. Advanced structural wind engineering. Springer, 2013.

[9] Earl Dowell. A Modern Course in Aeroelasticity. Springer International Publishing, 01 15.

[10] William H Press, Saul A Teukolsky, William T Vetterling, and Brian P Flannery. Numerical recipes in $\mathrm{c}++$. The art of scientific computing, 2:1002, 1992.

[11] Alan Garnett Davenport. The application of statistical concepts to the wind loading of structures. Proceedings of the Institution of Civil Engineers, 19(4):449-472, 1961.

[12] Alan G Davenport. The response of slender, line-like structures to a gusty wind. Proceedings of the Institution of Civil Engineers, 23(3):389-408, 1962. 
[13] Hans Wolfgang Liepmann. On the application of statistical concepts to the buffeting problem. Journal of the Aeronautical Sciences, 19(12):793-800, 1952.

[14] Vincent Denoël. On the background and biresonant components of the random response of single degree-of-freedom systems under non-gaussian random loading. Engineering structures, 33(8):2271-2283, 2011.

[15] Vincent Denoël and Luigi Carassale. Response of an oscillator to a random quadratic velocity-feedback loading. Journal of Wind Engineering and Industrial Aerodynamics, 147:330-344, 2015.

[16] Vincent Denoël. Multiple timescale spectral analysis. Probabilistic Engineering Mechanics, 39:69-86, 2015.

[17] Julien Heremans, Anass Mayou, and Vincent Denoël. Background/resonant decomposition of the stochastic torsional flutter response of an aeroelastic oscillator under buffeting loads. Journal of Wind Engineering and Industrial Aerodynamics, 208:104423, 2021.

[18] Anurag Jain, Nicholas P Jones, and Robert H Scanlan. Coupled flutter and buffeting analysis of long-span bridges. Journal of Structural Engineering, 122(7):716-725, 1996.

[19] H Katsuchi, NP Jones, RH Scanlan, and H Akiyama. Multi-mode flutter and buffeting analysis of the akashi-kaikyo bridge. Journal of Wind Engineering and Industrial Aerodynamics, 77:431-441, 1998.

[20] YJ Ge and H Tanaka. Aerodynamic flutter analysis of cable-supported bridges by multimode and full-mode approaches. Journal of Wind Engineering and Industrial Aerodynamics, 86(2-3):123-153, 2000.

[21] Tommaso Argentini, A Pagani, Daniele Rocchi, and Alberto Zasso. Monte carlo analysis of total damping and flutter speed of a long span bridge: Effects of structural and aerodynamic uncertainties. Journal of Wind Engineering and Industrial Aerodynamics, 128:90-104, 2014.

[22] Thomas Canor, Luca Caracoglia, and Vincent Denoël. Application of random eigenvalue analysis to assess bridge flutter probability. Journal of Wind Engineering and Industrial Aerodynamics, 140:79-86, 2015.

[23] Vincent Denoël. Estimation of modal correlation coefficients from background and resonant responses. Structural Engineering and Mechanics: an International Journal, 32(6):725-740, 2009.

[24] Vincent Denoël and Hervé Degée. Asymptotic expansion of slightly coupled modal dynamic transfer functions. Journal of Sound and Vibration, 328(1-2):1-8, 2009.

\section{APPENDIX: THE ADAPTATIVE INTEGRATION SCHEME}

The results obtained and presented in this paper are partly obtained with a simple in-house adaptative integration scheme. The algorithm works as follows. First, the domain of integration is divided into segments separated by the integration points. On each segment, the contribution to the integral is computed by means of the trapezoidal rule. These segments are then iteratively refined, if required, until a desired accuracy is reached. 
The refinement procedure consists in adding an integration point in the middle of the interval, creating therefore two sub-segments instead of the initial one. The sum of the contribution on the two sub-segments is compared to the contribution along the domain before subdivision. If the relative difference between both is lower than a user-defined threshold, then this domain in not subdivided anymore. This process is continued until no segments requires being divided anymore.

This algorithm performs better when the original distribution of points includes the inflexion points in the function to integrate. This is due to the fact that the break-down condition originates from a concept built on a curvature with a constant sign along the domain. The termination criteria can indeed be too confident in case of a domain with an inflexion point in the middle.

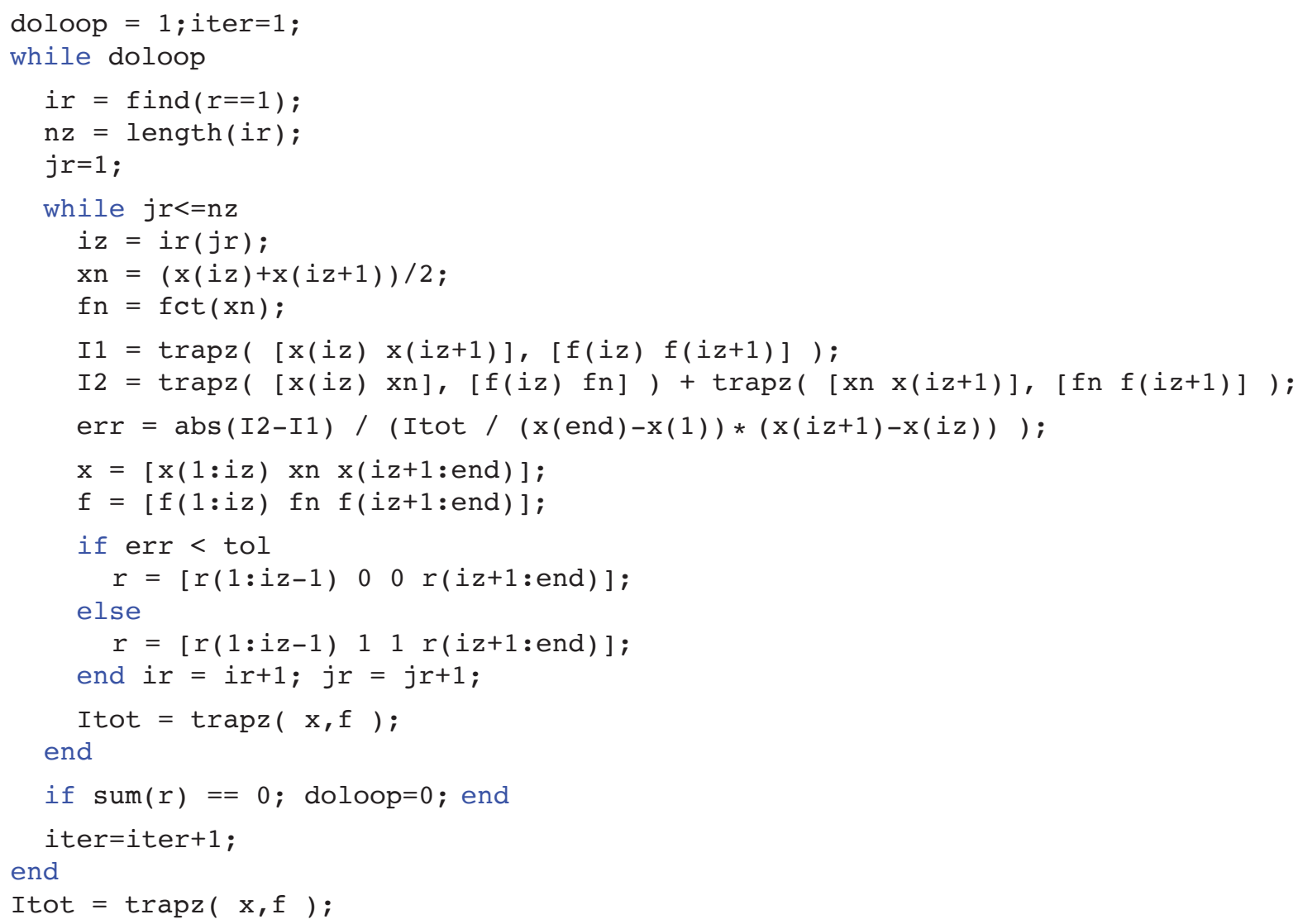

